\title{
Design of a MEMS speaker driver
}

\author{
M. Hänsler, D. Strle, M. Auer OVE
}

Microelectromechanical systems (MEMS) have already found widespread use in highly integrated audio applications. Currently, they are used for microphones where their small size has enabled the use of multiple microphones in a single device to improve noise cancellation. Nowaday, even loudspeakers realized as MEMS structures are possible, which brings up new challenges in circuit design that are the focus of this paper.

Starting with an overview of current research activities of MEMS speakers, their advantages and disadvantages, as well as the peculiarities of the driver design are discussed. Compared to electrodynamic speakers, MEMS speakers have a different physical structure which needs to be accounted for when designing the driving circuitry. Possible driver concepts are presented and compared with special consideration of their efficiency and audio quality. Existing concepts are shown and new concepts are proposed.

Keywords: MEMS; MEMS speaker; MEMS driver; audio amplifier; high efficient; energy recovery

\section{Design eines Treibers für MEMS-Lautsprecher.}

Mikroelektromechanische Systeme (MEMS) haben bereits eine weite Verbreitung in hoch integrierten Audioanwendungen gefunden. Aktuell werden sie für Mikrofone verwendet, wo durch ihre geringe Bauform die Möglichkeit geschaffen wurde, mehrere Mikrofone in einem einzelnen Gerät zu verbauen und dadurch die Geräuschunterdrückung zu verbessern. Heutzutage sind jedoch auch schon Lautsprecher als MEMS-Strukturen realisierbar. Dies hat neue Herausforderungen im Bereich der Schaltungstechnik zur Folge, welche den Kern dieses Artikels bilden.

Beginnend mit einem Überblick über aktuelle Forschungsaktivitäten von MEMS-Lautsprechern werden deren Vor- und Nachteile sowie im Speziellen deren Eigenschaften bezüglich des benötigten Treibers diskutiert. Verglichen mit elektrodynamischen Lautsprechern haben MEMS-Lautsprecher eine unterschiedliche physikalische Struktur, auf welche beim Schaltungsdesign geachtet werden muss. In dieser Arbeit werden mögliche Treiberkonzepte präsentiert und speziell in Bezug auf deren Effizienz und Audioqualität verglichen. Bereits vorhanden Konzepte werden erläutert sowie ein neues Konzept vorgestellt.

Schlüsse/wörter: MEMS-Lautsprecher; MEMS-Treiber; Audioverstärker; hocheffizient; Energierückgewinnung

\section{Introduction}

Microelectromechanical systems (MEMS) are becoming increasingly important in various industrial areas, were they are used in a wide range of applications like sensors, oscillators or microphones [1]. The improvements in MEMS technologies open up new application areas where MEMS devices can be used. One of these new possibilities are miniaturized MEMS speakers.

In the following the most important fundamentals of MEMS speakers, their requirements on the driving circuitry as well as an overview about existing speaker amplifier architectures and their advantages/disadvantages together with MEMS speakers is given. Commonly used driving architectures for mobile applications are discussed with the focus on driving capacitive loads. A New architecture for MEMS speaker drivers is introduced and first simulation as well as measurement results of the first prototypes are presented.

\section{MEMS speaker fundamentals}

The principle of a MEMS based speaker is shown in Fig. 1. An electric field is applied to a piezoelectric cantilever, that consists of two layers. One side is fixed which results in a displacement of $\Delta \mathrm{X}_{\text {Out }}$ of the opposite side. This mechanical movement can be used to control an attached membrane to generate the acoustic output of the speaker. The movement depends on the polarization of the material as well as the applied voltage. As depicted in Fig. 1, the top and bottom plates have different polarizations which causes one layer

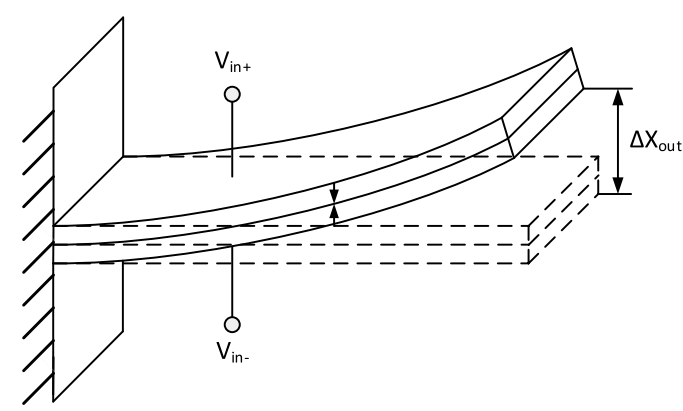

Fig. 1. Piezoelectric actuator before (dashed) and after (solid) applying a voltage. The left side of the actuator is fixed and the right side deflects by $\Delta \mathbf{X}_{\text {out }}$

to expand and the other layer to contract. The advantage of such a multi layer piezoelectric cantilever is the reduction of the required voltage [2]

Hänsler, Markus, Institute of Electronics, Graz University of Technology, Inffeldgasse 12//, 8010 Graz, Austria (E-mail: markus.haensler@gmail.com); Strle, Drago, Faculty of Electrical Engineering University of Ljubljana, Tržaška cesta 25, 1000 Ljubljana, Slovenia; Auer, Mario, Institute of Electronics, Graz University of Technology, Inffeldgasse 12/l, 8010 Graz, Austria 


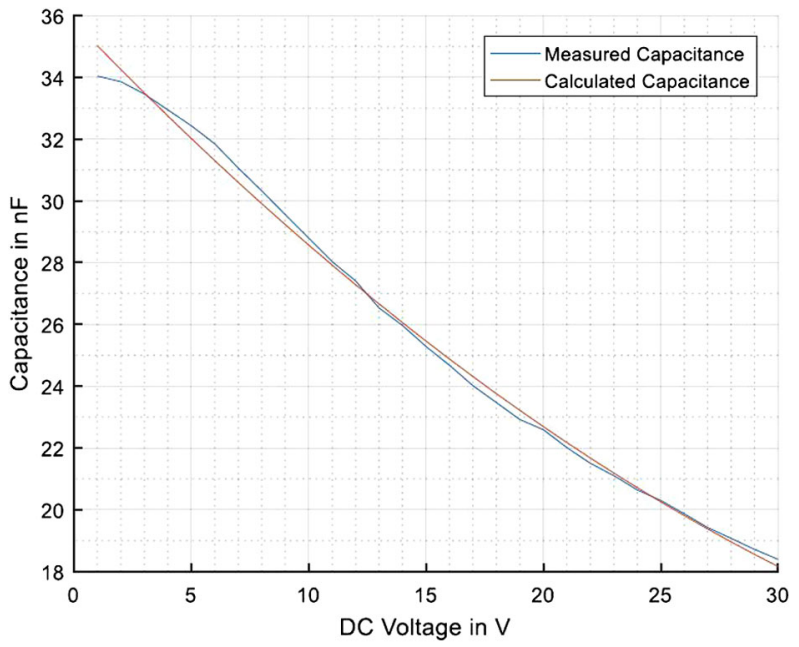

Fig. 2. Comparison between measurement results of the piezoelectric device and the second order capacitance model. The applied signal amplitude was $1 \mathrm{~V}$ peak to peak $\mathrm{AC}$ with varying $\mathrm{DC}$ offset voltages from $1 \mathrm{~V}$ to $30 \mathrm{~V}$ at a frequency of $10 \mathrm{kHz}$

One of the biggest advantages of MEMS speakers compared to electrodynamic speakers is the fact that the electrical equivalent circuit of a MEMS speaker can be modelled in a first approximation as a capacitor. This allows different driving architectures but on the other hand results also in new challenges for the driver circuit. The most important considerations will be discussed in the following chapters.

\subsection{Non-linearity}

One of the biggest challenges for MEMS speaker drivers is the nonlinear behavior of the speaker's capacitance. Piezoelectric materials have a non-linear dielectric constant which results in a capacitance of the speaker that depends on the voltage as well as the displacement of the membrane. This behavior is already well known and for example described in $[3,4]$. The results of these investigations can be used for a polynomial approximation of this behavior. A second order polynomial is used to model the capacitance as a function of the applied voltage

$$
C_{\text {Speaker }}=\frac{1}{C_{n}}\left(P_{0, n}+P_{1, n} \cdot V_{D C}+P_{2, n} \cdot V_{D C}^{2}\right)
$$

where $C_{\text {Speaker }}$ is the non-linear speaker capacitance, $C_{n}$ is the nominal capacitance of the speaker, $P_{\mathrm{i}, \mathrm{n}}$ are the non-linear coefficients and $V_{D C}$ is the $D C$ voltage at the speaker. The difference between the measured non-linearity and the approximation can be seen inside Fig. 2 for a $1 \mathrm{~V}$ peak-to-peak $A C$ signal and different DC offset voltages at $10 \mathrm{kHz}$. It can be seen that the slope is approximately constant and only drops at low DC offset voltages. To avoid that the polarity of the voltage across the piezoelectric material changes, the speaker requires a constant DC offset which will be set to the middle of the maximum required output voltage. To achieve $30 V_{P P}$ at the speaker, a minimum $D C$ offset of $15 \mathrm{~V}$ is required.

It can be seen, that the non-linearity is not only depending on the DC voltage, but is also varying over the audio frequency range. Figure 3 shows this effect based on measurements results. Compared to the voltage depending non-linearity, this effect is considered to be small and won't be considered for first driver designs.

\subsection{Power factor}

The fact that a MEMS speakers present a capacitive load to the driver results in a difference in the power factor $\cos (\phi)$ compared to con-

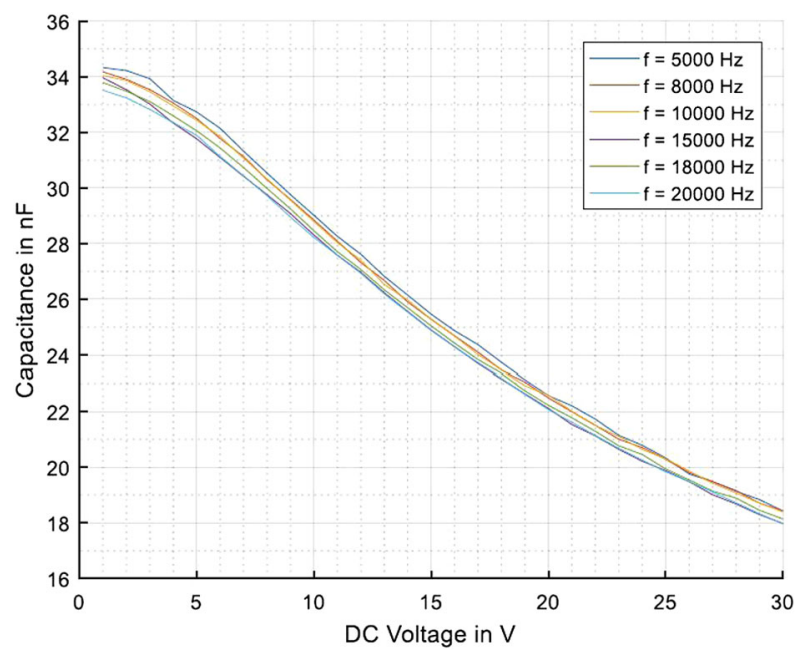

Fig. 3. Frequency dependency of the non linear capacitance of the speaker. The applied signal had an amplitude of $1 \mathrm{~V}$ peak to peak $A C$ with varying $D C$ offset voltages from $1 \mathrm{~V}$ to $30 \mathrm{~V}$ and varying frequencies from $5 \mathrm{kHz}$ to $20 \mathrm{kHz}$

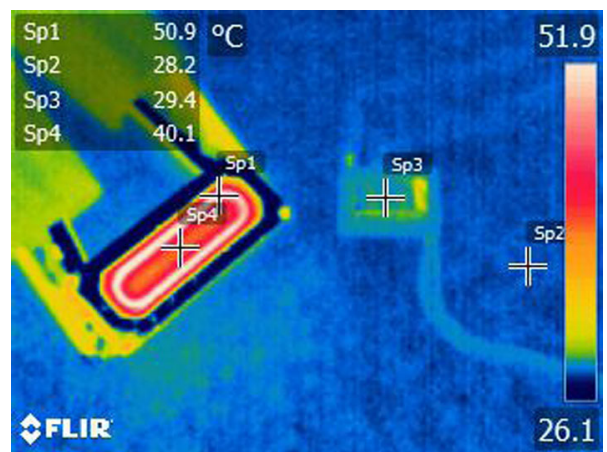

Fig. 4. Comparison of the self-heating for different speaker types. Sp1 is a traditional electrodynamic speaker and Sp3 a MEMS based speaker. Measurement point Sp2 is the temperature of the environment for comparison

ventional electrodynamic speakers. The power factor describes the ratio of real power $P$ to apparent power $S$

$$
\cos (\phi)=\frac{P}{|S|}
$$

where $\phi$ is the phase shift between the voltage and current. This factor can be in the range of $0^{\circ}$ to $45^{\circ}$ for electrodynamic or balanced armature speakers. This results in a considerable amount of real power which causes a temperature increase at the speaker. Compared to this value, the power factor for MEMS speakers is in the range of $80^{\circ}$ to $90^{\circ}$ which shows that the real power losses inside the speaker are very low compared to electrodynamic speakers. This results in a much lower temperature increase of the speaker which can be seen in Fig. 4. Compared to the electrodynamic speaker which reaches $40.1^{\circ} \mathrm{C}$ at the membrane and $50.9^{\circ} \mathrm{C}$ at the coil, the temperature of the MEMS speaker is with $29.4^{\circ} \mathrm{C}$ only slightly higher than the desk on which they were placed.

\subsection{Hysteresis}

A third important parameter of MEMS speakers is the hysteresis effect of the speaker. Figure 5 shows the hysteresis of the speaker as 
a function of the applied voltage and the resulting current into the speaker. This effect is dependent on the type of driving architecture used, as described in $[5,6]$ and is considered inside for the driving concept as well.

\section{State of the art audio amplifiers}

Currently many designs for mobile applications are based on either Class D or Class $\mathrm{H}$ amplifiers. Therefore their key parameters were analyzed and a comparison was made regarding their capabilities for driving electrodynamic and piezoelectric MEMS speakers.

\subsection{Class D amplifier}

Class D amplifiers are very often used inside low power applications. To analyze the impact of the capacitive load on the amplifier, only the output stage will be considered. Figure 6 shows the block diagram of a typical Class D amplifier architecture.

A key parameter for mobile audio applications is the efficiency of the used amplifier. This is also a point where the biggest impact of the different load can be seen and new considerations have to be taken. The efficiency of audio amplifiers for electrodynamic speaker is often calculated based on the average power at the output [7]

$$
P_{\text {Average }}=\frac{1}{T} \int_{0}^{T} u_{(\mathrm{t})} \cdot i_{(\mathrm{t})} \mathrm{d} t=V_{\mathrm{RMS}} \cdot I_{\mathrm{RMS}} \cdot \cos (\Phi),
$$

where $P_{\text {Average }}$ is the average active power, $T$ the period of the signal, $u_{(t)}$ the current value of the voltage, $i_{(t)}$ the current value of the

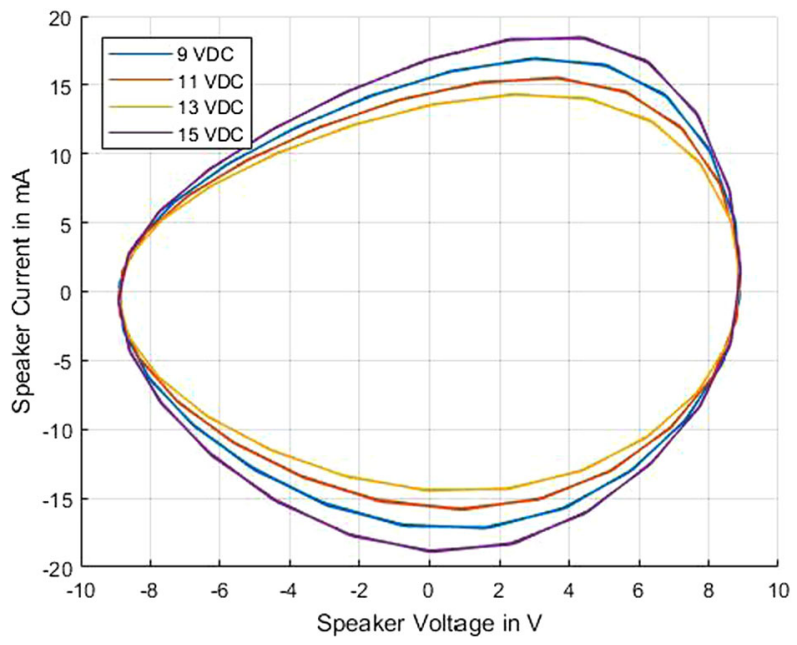

Fig. 5. Hysteresis effect of the speaker as a function of the applied voltage and the resulting current into the speaker. Measurement was done with different $\mathrm{DC}$ offset voltages an input signal of $9 \mathrm{~V}$ at $8 \mathrm{kHz}$ current, $V_{\text {RMS }}$ the root mean square (RMS) value of the voltage and $I_{\text {RMS }}$ the RMS value of the current. As shown above, the power factor for MEMS speakers is approaching zero which would result in a very low efficiency. For this reason the apparent power will be used to calculate the efficiency of amplifiers with capacitive loads [7].

$$
\eta=\frac{P_{\text {out }}}{P_{\text {out }}+P_{\text {diss }}},
$$

where $P_{\text {out }}$ is the apparent output power and $P_{\text {diss }}$ the amplifier dissipation. The losses of the amplifier can be separated into following parts [7]:

$$
P_{\text {diss }}=P_{\mathrm{Q}}+P_{\mathrm{CL}}+P_{\mathrm{SW}}+P_{\mathrm{BD}}+P_{\mathrm{Filt}},
$$

where $P_{\mathrm{Q}}$ is the quiescent power loss of the amplifier, $P_{\mathrm{CL}}$ the conduction losses of the output drivers, $P_{\mathrm{SW}}$ the switching losses, $P_{\mathrm{BD}}$ the bulk diode of the driving transistors and $P_{\text {Filt }}$ the losses inside the output filter and load. Considering the four parts of the power losses [7] calculated in (6) to (10)

$$
\begin{aligned}
P_{\mathrm{CL}}= & I_{\text {out }, \text { rms }} \cdot R_{\mathrm{ds}, \text { on }} \\
P_{\mathrm{SW}}= & \sum_{i} F_{\mathrm{SW}} \cdot V_{\mathrm{CP}}^{2} \cdot C_{\mathrm{P}, \mathrm{i}} \\
P_{\mathrm{BD}}= & V_{\mathrm{SD}} \cdot F_{\mathrm{SW}} \cdot\left(I_{\mathrm{pk}} \cdot t_{\mathrm{dt}}+I_{\mathrm{rrm}} \cdot t_{\mathrm{rr}}\right) \\
P_{\mathrm{FLLT}}= & I_{\text {out }, \text { rms }} \cdot\left|Z_{\mathrm{L}}\right| \cdot \cos (\Phi) \\
& +C_{\text {load }} \cdot V_{\text {out }, \text { rms }}^{2} \cdot 2 \cdot \pi \cdot f \cdot d f,
\end{aligned}
$$

where $l_{\text {out, }}$,ms is the output RMS current, $R_{\mathrm{ds}, \text { on }}$ is the drain source on resistance of the output transistor, $F_{S W}$ the switching frequency, $V_{C P}^{2}$ the voltage at each parasitic capacitance at the output, $C_{P, i}$ the parasitic capacitances at the output, $V_{S D}$ the body diode voltage drop, $I_{\mathrm{pk}}$ the peak output current, $t_{\mathrm{dt}}$ the dead time, $I_{\mathrm{rrm}}$ the body diode reverse recovery current, $t_{\mathrm{rr}}$ the reverse recovery time, $\left|Z_{\mathrm{L}}\right| \cdot \cos (\Phi)$ the resistive part of the output filter, $C_{\text {load }}$ the load capacitance and $f$ the applied frequency at the output, two major drawbacks of a Class D amplifier together with capacitive loads for ultra low power applications can be seen. First the conduction losses and the body diode losses are related to the output current (either rms or peak). Especially at high frequencies this current will be considerable high which causes high losses inside the output switches.

Second, the size of the inductor can be a critical parameter, especially for a small load capacitance as it is the case for MEMS speakers. Considering a load of $100 \mathrm{nF}$ and a cutoff frequency of $29 \mathrm{kHz}$ would results in an inductor value of approximately $300 \mu \mathrm{H}$ for the output filter.



Fig. 6. Block diagram of a typical Class D amplifier 


\subsection{Class $\mathrm{H}$ amplifier}

Class $\mathrm{H}$ amplifiers are a good alternative compared to Class D amplifiers in low power applications. Figure 7 shows the block diagram for a typical Class $\mathrm{H}$ amplifier. This type of amplifier is basically a class $A B$ architecture where the supply voltage of the output stage will be regulated based on the required output swing. The way the supply voltage is regulated is the main difference between a Class $G$ and a Class $\mathrm{H}$ amplifier. Figure 8 shows both supply voltage regulation topologies. Compared to Class $\mathrm{G}$ amplifiers, the supply voltage follows the signal continuously for a Class $\mathrm{H}$ Amplifiers whereas there are discrete supply voltages levels inside Class $\mathrm{G}$ amplifiers.

The efficiency of Class $\mathrm{H}$ amplifiers will be typically calculated in two different operating areas. The first area is where the input signal is small so that the adaptive supply voltage regulation is not working. In this region the Class $\mathrm{H}$ amplifier works like an Class $A B$ amplifier. For simplifications only this operating region will be analyzed for the efficiency calculations.

Considering a resistive load the maximum possible efficiency of a Class AB amplifier can be calculated as

$$
\eta_{\mathrm{R}}=\frac{P_{\text {In }}}{P_{\text {Out }}}=\frac{\pi}{4}=78.5 \%
$$

Considering a capacitive load, the resulting maximum possible efficiency reduces to

$$
\eta_{\mathrm{C}}=\frac{P_{\text {Out }}}{P_{\text {Out }}+P_{\text {Loss }}}=\frac{\pi}{\pi+4}=44.0 \%
$$

according to [8]. It can be seen that the efficiency will be dramatically reduced. Although power amplifiers with energy recovery functionality were proposed [9], they still require a charge pump which generates the high output voltage. Moreover, high voltage devices are required which increase the chip area and complexity of the design. Therefore, circuit architectures are required that work with a minimum number of high voltage devices and keep the chip area and the number of required external components at a minimum.

\section{Driver concepts}

One of the main challenges for MEMS speaker amplifiers is the required high voltage at the speaker. For the currently analyzed speakers the required voltage can go up to $30 \mathrm{Vpp}$ maximum. To be able to drive such types of speakers, typically a charge pump is required to supply the audio amplifier. It is therefore advantagous to combine those two circuit blocks to increase performance and efficiency of audio amplifiers for MEMS speakers.

One additional benefit of the MEMS speaker technology is the fact that the speaker mainly consumes reactive power. With proper system architecture this energy can be recovered to significantly increase the efficiency of the overall system. Energy transfer from or to a capacitor can be done either using a capacitive approach or based on an LC resonant circuit. Both of these architectures will be explored in the following sections. The energy transfer should be done in a way to generate an output voltage that is higher than the input voltage, i.e. to boost the voltage. The minimum ratio of 10 should be achieved since the speaker will be driven with a signal amplitude of $30 \mathrm{Vpp}$.

\subsection{Capacitive based}

One of the most basic circuits for capacitive energy transfer is the switched capacitor voltage doubler [10]. The basic circuit diagram of a switched capacitor voltage doubler can be found in Fig. 9. Four stages are required to generate an input voltage to output voltage ratio of at least 10 . The efficiency of the circuit is one of the most important parameters. For this reason the energy loss caused by connecting two capacitors in parallel is calculated.

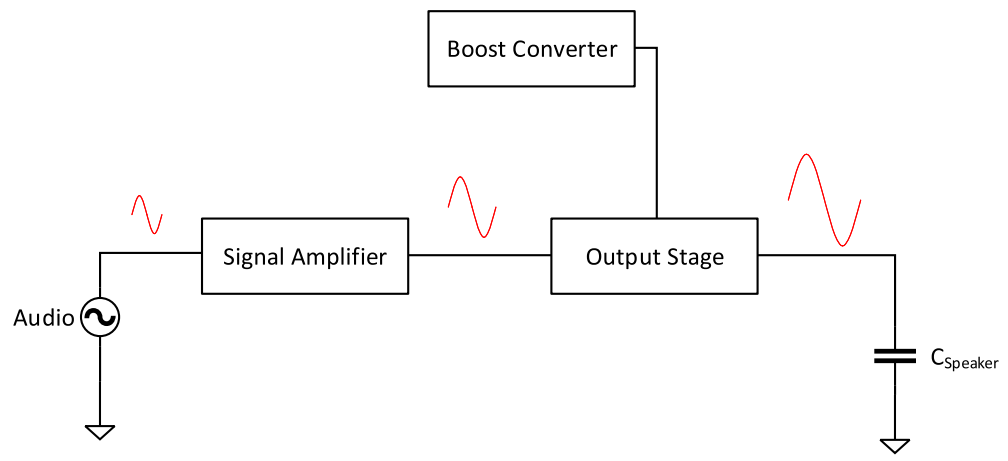

Fig. 7. Block diagram for a Class $\mathrm{H}$ amplifier showing the signal amplifier, the Boost converter and the output stage
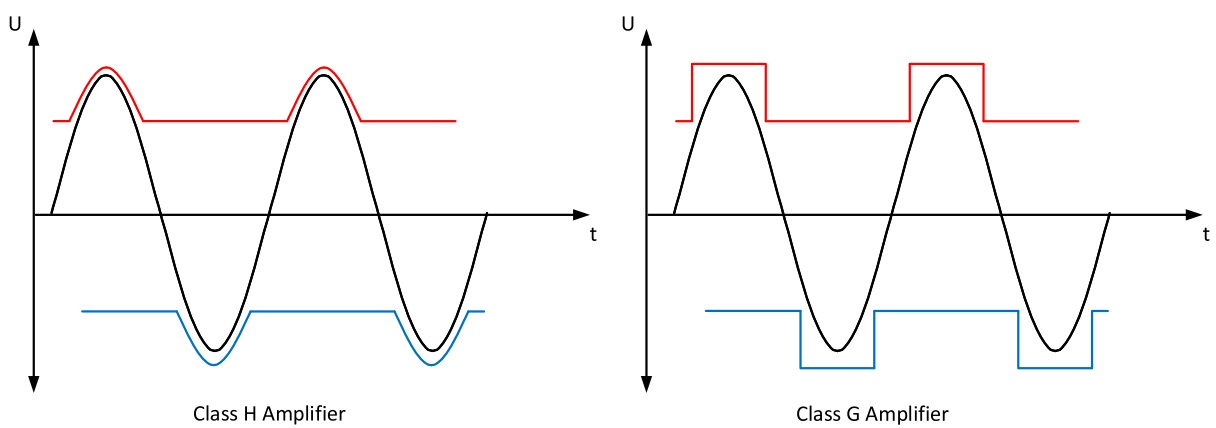

Fig. 8. Output signals and supply voltages for Class $\mathrm{H}$ and Class $\mathrm{G}$ amplifiers 


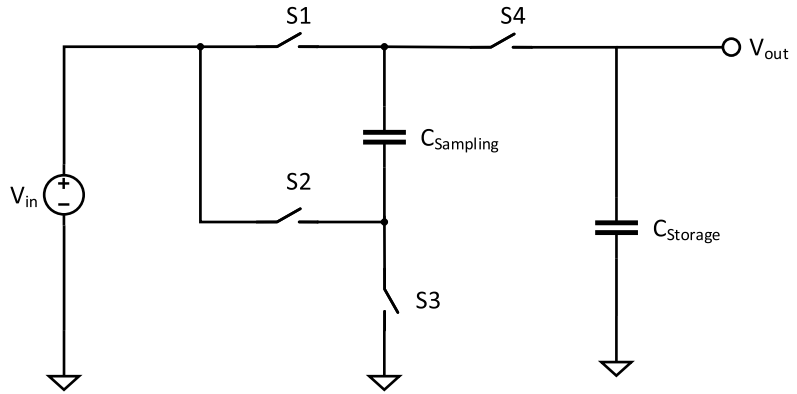

Fig. 9. Basic circuit for a switched capacitor voltage doubler including the four switches as well as the sampling capacitor $C_{\text {Sampling }}$ and the storage capacitor $C_{\text {storage }}$

Connecting two capacitors in parallel results in a common voltage $V_{\text {common }}$ which can be calculated as

$$
\begin{aligned}
Q_{\text {ges }} & =C_{1} \cdot V_{1}+C_{2} \cdot V_{2} \\
\left(C_{1}+C_{2}\right) \cdot V_{\text {common }} & =C_{1} \cdot V_{1}+C_{2} \cdot V_{2} \\
V_{\text {common }} & =\frac{C_{1} \cdot V_{1}+C_{2} \cdot V_{2}}{C_{1}+C_{2}},
\end{aligned}
$$

where $Q_{\text {ges }}$ is the total charge inside the system, $C_{1}$ is the first capacitor, $V_{1}$ the voltage at the first capacitor, $C_{2}$ is the second capacitor and $V_{2}$ the voltage at the second capacitor, which results in an energy $E_{\text {common }}$ inside the system after charge transfer of

$$
\begin{aligned}
E_{\text {common }} & =\frac{C_{\text {ges }} \cdot V_{\text {common }}^{2}}{2} \\
& =\frac{1}{2}\left(C_{1}+C_{2}\right)\left(\frac{C_{1} \cdot V_{1}+C_{2} \cdot V_{2}}{C_{1}+C_{2}}\right)^{2} .
\end{aligned}
$$

Based on this, the energy loss $\Delta E$ during the charge transfer can be calculated. The initial energy $E_{0}$ inside the system can be written as

$$
E_{0}=\frac{1}{2} C_{1} \cdot V_{1}^{2}+\frac{1}{2} C_{2} \cdot V_{2}^{2}
$$

which results in an energy loss off

$$
\begin{aligned}
\Delta E= & E_{0}-E_{\text {common }} \\
= & \frac{1}{2} C_{1} \cdot V_{1}^{2}+\frac{1}{2} C_{2} \cdot V_{2}^{2} \\
& -\frac{1}{2}\left(C_{1}+C_{2}\right)\left(\frac{C_{1} \cdot V_{1}+C_{2} \cdot V_{2}}{C_{1}+C_{2}}\right)^{2} \\
= & \frac{1}{2} \frac{C_{1} \cdot C_{2}}{C_{1}+C_{2}}\left(V_{1}-V_{2}\right)^{2} .
\end{aligned}
$$

It can be seen that there is always an energy loss once charge is transferred from one capacitor to another. Assuming two identical capacitors this results in the fact that the output voltage will be half of the input voltage and output energy which is only a quarter of the input energy. This is a major drawback of a capacitive based solution. Nevertheless it is also important to notice that the energy loss is not depending on the switch on resistance.

The second major drawback of this solution will become obvious once charge recovery structures will be considered. In a typical voltage doubler or any charge pump circuit, the energy transfer goes from the input to the output of the voltage doubler. This is not the case if energy recovery will be considered. The basic block diagram of such an architecture is shown inside Fig. 10.

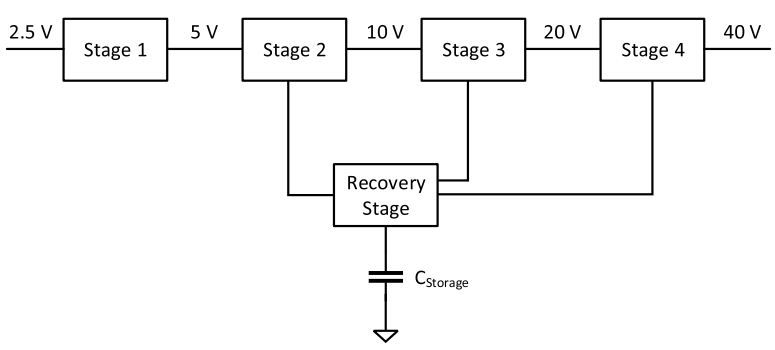

Fig. 10. Basic circuit block diagram for a capacitive based architecture including four switched capacitor voltage doubler stages and the main recovery stage for charge recovery

In such an architecture, the current inside the output stages (at least in the third and fourth stage) is bidirectional and can flow towards the load but also from the load towards the internal storage capacitor. This requires symmetrical high voltage switches inside these stages, which limits the number of available process technologies. Also the capacitors required for the voltage doublers can be in the range of up to $100 \mathrm{nF}$ for the first stages (due to the high reactive power which is required at the output) which would require either a big silicon area or additional external components.

\subsection{Inductor based}

An alternative approach to the capacitor based solutions are inductor based architectures. The first applications for such architectures were plasma panels where the fundamentals of inductor based charge recovery were developed and patented [11]. The basic structure of such an energy recovery system can be seen in Fig. 11 .

These architectures work on the principle where energy will be transferred either from the supply or a storage capacitor to the load (typically a capacitor or device with capacitive behavior) using an inductor.

Nowadays these types of architectures are again becoming important especially for applications like piezoelectric actuators where also high voltages are required and the load can be considered as a capacitive load [13]. Figure 12 shows such a circuit which is used to drive a piezoelectric actuator

Most of the circuits which include energy recovery as well are used to generate rectangular output signals. These types of applications are working typically with very high voltages and the requirements on the signal quality are usually lower.

\subsection{Proposed architecture}

One disadvantage of all previously discussed architectures is the fact that they need a separate boost converter to generate the high output voltage at the driver stage, where also the generation of the additional required DC offset has to be considered. New solutions should be aimed at the development of new driver architectures, that combine the amplifier and the boost converter stage in an efficient way and to use energy recovery concepts without having a significant impact on the signal quality.

Based on solutions for the generation of rectangular output signals, the transition to high quality audio signals with low THD has to be made. Because of the high output voltage, also large signal parameters like the slew rate have to be observed.

The voltage at the load can be expressed as

$$
v_{\text {load }}=V_{D C}+V_{P} \cdot \sin \left(2 \cdot \pi \cdot f_{\max } \cdot t\right),
$$

where $v_{\text {load }}$ is the voltage at the speaker, $V_{D C}$ is the $D C$ voltage at the speaker, $V_{\mathrm{P}}$ is the peak $\mathrm{AC}$ voltage at the speaker and $f_{\max }$ the 


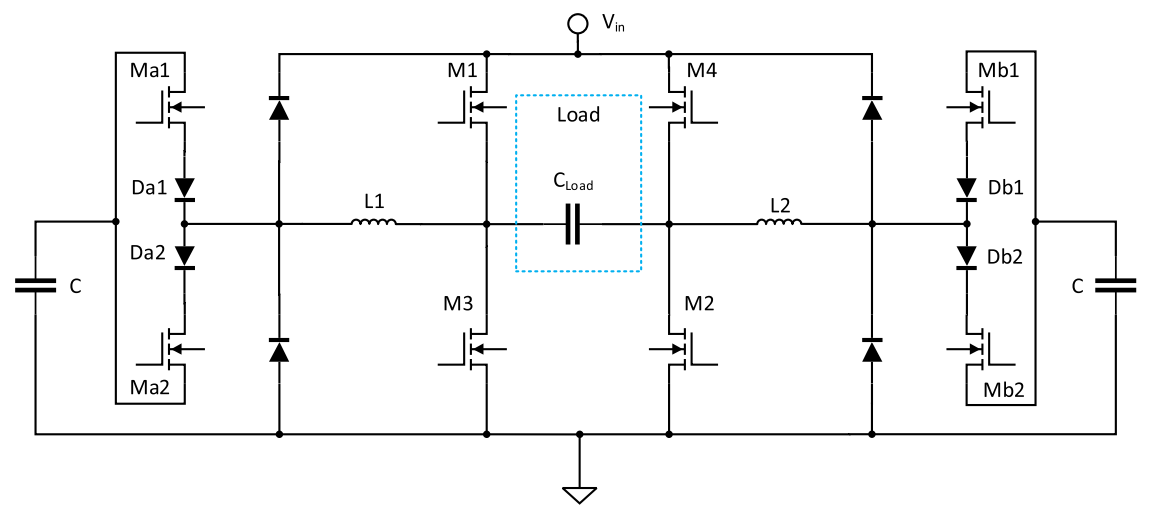

Fig. 11. Full bridge inverter [12] based on Weber circuit. The dedicated switching signals for each transistor are not drawn explicitly

maximum possible output signal frequency. This results in a required slew rate of

$$
\frac{\partial v_{\text {load }}}{\partial t}=V_{p} \cdot 2 \cdot \pi \cdot f_{\max } \cdot \cos \left(2 \cdot \pi \cdot f_{\max } \cdot t\right),
$$

which is for an audio frequency up to $20 \mathrm{kHz}$ with maximum amplitudes of $30 \mathrm{~V}$ peak to peak in the range of $1.885 \mathrm{~V} / \mu \mathrm{s}$. This high slew rate is also the reason why currently available audio amplifiers for ceramic speakers are limiting the output voltage swing for frequencies higher than approximately $10 \mathrm{kHz}$.

Figure 13 shows the basic block diagram of the proposed solution The core of the circuit is the HV driver stage which is similar to the one shown inside Fig. 12. The advantage of this architecture is the fact that no additional boost converter is required and the high output voltage is directly generated at the load. Therefore, with small modifications on the output stage, only two devices have to be high voltage devices.

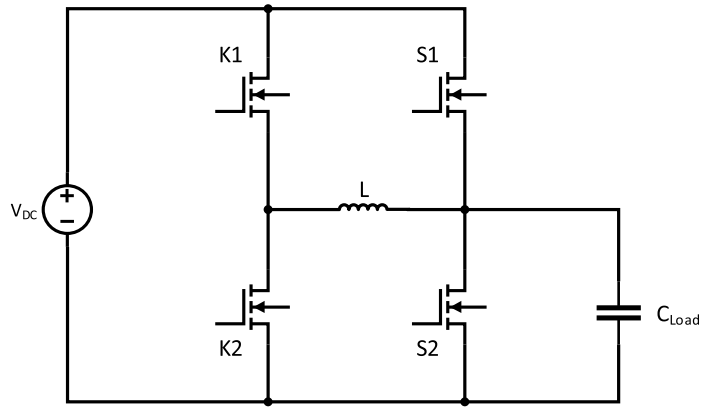

Fig. 12. Inductor based driver for piezoelectric actuator. The dedicated switching signals for each transistor are not drawn explicitly
Since the high voltage is generated directly at the load, this node is very sensitive related to currents drawn from the load. Therefore special considerations have to be taken into account to design the voltage divider, which is required to keep the voltage of the $A D C$ input stage in the allowed range for low voltage devices.

Another important design aspect is the switching of the output driver. This has to be done in a way to keep on one side the output transistors on resistance as low as possible and on the other hand to avoid any unwanted oscillation on the output which would decrease the system efficiency.

To be able to generate a sine wave output signal, a feedback loop is introduced including a voltage divider and analog to digital converter. This feedback provides the information for the digital controller to be able to drive the boost energy-recovery stage and at the same time eliminating non-linear effects from the speaker.

To be able to control the HV stage, an accurate PWM signal is required to control the driver switches. Based on this PWM signal, the voltage steps at the output of the amplifier can be controlled which allows an accurate control of the output voltage and therefore an increased signal quality. The control of the system should also be able to support the high dynamic range of the audio signals. A human ear has a dynamic range of $130 \mathrm{~dB}$ which should be considered during the design of the control loop. Therefore a trade off between speed (required for high frequency signals with high amplitude) and signal quality at lower amplitudes has to be found.

4.4 Simulation and measurement results

The simulations were done based on a MATLAB/Simulink model of the whole system including non linear effects of the speaker. Figure 14 shows the first results based on a $5 \mathrm{kHz}$ input signal. A total harmonic distortion of $-78 \mathrm{~dB}$ and a SNR of $86 \mathrm{~dB}$ could be achieved at $15 \mathrm{Vp}$ output amplitude.

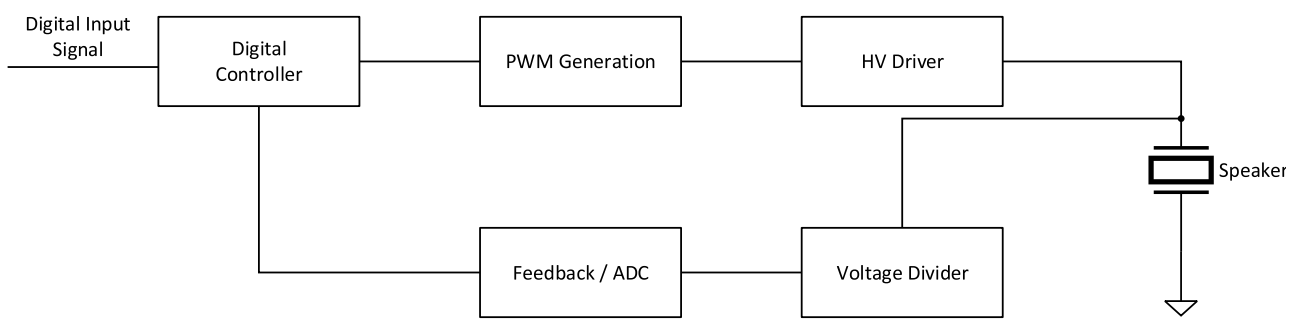

Fig. 13. Proposed inductor based driver for piezoelectric MEMS speakers 


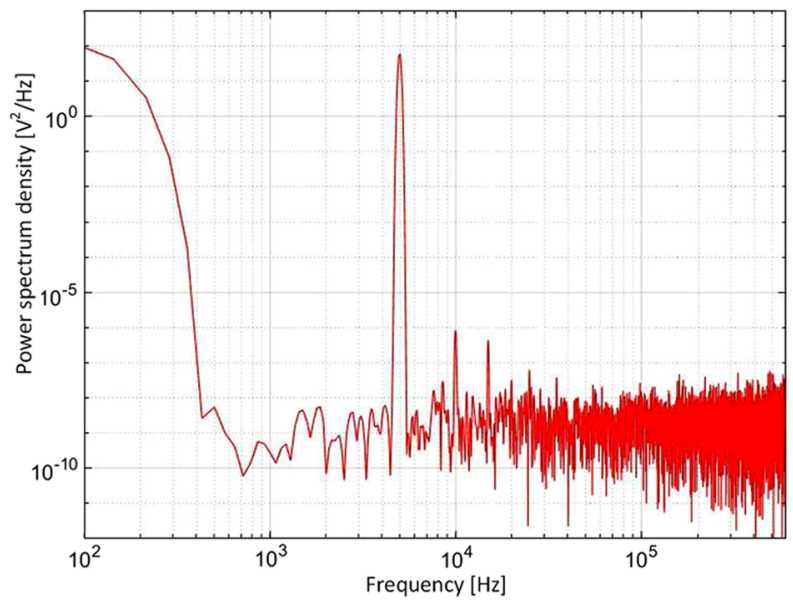

Fig. 14. Matlab simulation results for the proposed circuit including main losses (like switching losses or diodes). Simulation done with $15 \mathrm{~V}$ and a frequency of $5 \mathrm{kHz}$ at the speaker. An SNR of $86 \mathrm{~dB}$ and harmonic distortion of $-78 \mathrm{~dB}$ could be achieved

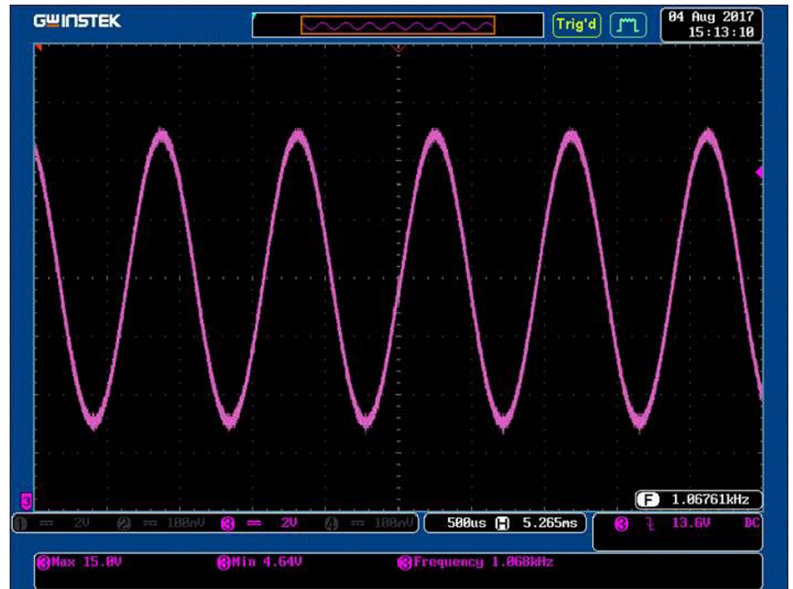

Fig. 15. First measurements based on an 0.18 XFAB SOI HV driving stage and FPGA for control loop

Also the energy recovery efficiency was analyzed using simulations. The energy consumed by the amplifier could be reduced by $67 \%$ including energy recovery compared to the same driving circuit but without energy recovery.

The first measurement results based on an $\mathrm{HV}$ driver fabricated in a $0.18 \mu \mathrm{m}$ XFAB SOI HV process and FPGA for the control loop. The results of the measurement can be seen in Fig. 15.

The currently maximum achievable SNR based on measurements is approximately $60 \mathrm{~dB}$. The low SNR is mainly related to the used analog circuitry on the $\mathrm{PCB}$ to divide the high output voltage to a lower level for the $A D C$ input. This currently limits the overall accuracy of the $A D C$ to approx. 8 bit and needs to be improved.

\section{Summary and conclusion}

Piezoelectric actuators are becoming increasingly important which brings again systems including energy recovery into the focus of research activities. Compared to actuators and plasma display panels, where up to now most of the research activities in terms of energy recovery were done, the signal quality for audio applications is one of the most important parameters. Nevertheless, the efficiency should still be considered. The proposed solution shows already good results for the energy recovery efficiency and also demonstrated the ability of the system to generate sinusoidal output signals. Future improvements have to be done to further increase the signal quality of the output signal as well as the efficiency of the energy recovery.

One major drawback of the inductor based energy recovery circuits is the strong dependency on the load capacitance and the inductance of the connection to the load. This results in ringing of the output signal during switching activities and an increase of THD. This impact has to be analyzed as well as optimized to be less sensitive on load variations and load conditions.

\section{Acknowledgements}

Open access funding provided by Graz University of Technology.

Open Access This article is distributed under the terms of the Creative Commons Attribution 4.0 International License (http://creativecommons.org/ licenses/by/4.0/), which permits unrestricted use, distribution, and reproduction in any medium, provided you give appropriate credit to the original author(s) and the source, provide a link to the Creative Commons license, and indicate if changes were made.

\section{References}

1. Yole Developpement (2017): Press releases gallery-MEMS [Online]. Available: https://www.i-micronews.com/images/Flyers/MEMS/YDMS17022_Status_of_the_ MEMS_industry_2017_flyer.pdf (visited on 08/07/2017)

2. I. Piezo Systems (2011): Introduction to piezo transducers [Online]. Available: http://www.piezo.com/catalog8.pdf\%20files/Cat8.22,23,24,25,62,63\&64.pdf.

3. Perez, R., Garcia, J. E., Albareda, A. (2000): Nonlinear dielectric behavior of piezoelectric ceramics. In ISAF 2000. Proceedings of the 2000 12th IEEE international symposium on applications of ferroelectrics (IEEE Cat. no. 00CH37076) (Vol. 1, pp. 443446). https://doi.org/10.1109/ISAF.2000.941592.

4. Rahman, M. A., Mamun, A. A. (2014): Nonlinearity analysis, modeling and compensation in PZT micro actuator of dual-stage actuator system. In 11th IEEE international conference on control automation (ICCA) (pp. 1275-1280). https://doi.org/ 10.1109/ICCA.2014.6871107.

5. Fleming, A. J. (2013): Precision charge drive with low frequency voltage feedback for linearization of piezoelectric hysteresis. In 2013 American control conference (pp. 6022-6026). https://doi.org/10.1109/ACC.2013. 6580781.

6. Rios, S. A., Fleming, A. J. (2016): Design of a charge drive for reducing hysteresis in a piezoelectric bimorph actuator. IEEE/ASME Trans. Mechatron., 21(1), 51-54. https://doi.org/10.1109/TMECH.2015.2483739. ISSN:1083-4435.

7. Colli-Menchi, A. I., Sanchez-Sinencio, E. (2015): A high-efficiency self-oscillating class-D amplifier for piezoelectric speakers. IEEE Trans. Power Electron., 30(9), 51255135. https://doi.org/10.1109/TPEL.2014.2363406. ISSN:0885-8993.

8. Nielsen, D. (2014): Class D audio amplifiers for high voltage capacitive transducers. PhD thesis, Technical University of Denmark.

9. Liang, H., Jiao, Z., Zhang, R., Wang, X., Liu, X. (2011): Design of a power amplifer with energy recovery strategy for piezoelectric actuators. In Proceedings of 2011 international conference on fluid power and mechatronics (pp. 207-212). https://doi.org/ 10.1109/FPM.2011.6045758

10. Dickson, J. F. (1976): On-chip high-voltage generation in MNOS integrated circuits using an improved voltage multiplier technique. IEEE J. Solid-State Circuits, 11(3), 374-378. https://doi.org/10.1109/JSSC. 1976.1050739. ISSN:0018-9200.

11. Weber, L. F., Warren, K. W., Wood, M. B. (1992): Power efficient sustain drivers and address drivers for plasma panel. US Patent 5,081,400.

12. Kim, C. J., Park, J., Kim, E. S. (2010): A study on the energy recovery circuit design for energy efficient driving of plasma flat lamp(PFL). In 2010 international conference on electrical machines and systems (pp. 1948-1952).

13. Vasic, D., Costa, F. (2014): Energy recovery power supply for piezoelectric actuator. In IECON 2014-40th annual conference of the IEEE industrial electronics society (pp. 1440-1445). https://doi.org/10.1109/IECON.2014.7048691. 


\section{Authors}



\section{Markus Hänsle}

received the B.Sc. and M.Sc. in electronics with focus on microelectronic and circuit design at the Graz University of Technology, Graz, Austria, 2013 and 2015, respectively, where he was working on RFID systems and design of ultra-low power current sensors. Currently, he is working toward the Ph.D. degree. His research interests are in the fields of high efficient low power audio amplifiers for microelectromechanical systems (MEMS) speakers. Currently, he is a design engineer with USound GmbH, Graz, Austria.

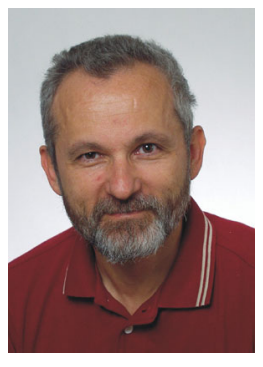

\section{Drago Strle}

received the Ph.D. degree from the University of Ljubljana, Faculty of Electrical Engineering, Ljubljana, Slovenia, in the field of synthesis of switched capacitor circuits in 1991. He has more than 30 years of experience in the field of design of analog and mixed signal ICS, especially low-noise and high-performance sensor interface circuits. Currently, he is a professor at the University of Ljubljana, teaching microelectronic courses. His research interests focus on but are not limited to the design and test of mixed-signal integrated circuits, analog and digital signal processing, modeling and design of highperformance interface circuits for MEMS, chemical and biological sensors.



\section{Mario Auer}

is Assistant Professor at the Institute of Electronics, Graz University of Technology, Austria. He is working in the field of microelectronics, integrated analog circuit design and electromagnetic compatibility. His research interests include the design of mixed-signal circuits ranging from applications for consumer electronics to radiation hard blocks. 